\title{
Tropheryma whipplei in the stool samples of children with acute diarrhea: a study from Tehran, Iran
}

\author{
Shirin Sayyahfar ${ }^{1}$, Mina Latifian ${ }^{2,3}$, Parisa Esmaeilii, ${ }^{2,3}$, Neda Baseri $^{2,3}$, Fahimeh Bagheri Amiri $^{3}$, Bita Bakhshi $^{4}$,
} Abdoulreza Esteghamati ${ }^{1}$ and Saber Esmaeili ${ }^{2,3^{*}}$

\begin{abstract}
Background: Recently, Tropheryma whipplei has been suggested as one of the causative agents of diarrhea among children worldwide. Limited data is available on the prevalence of T. whipplei among children with diarrhea in most countries such as Iran. This study was conducted to evaluate the prevalence of T. whipplei in children with acute diarrhea in Iran.

Methods: In this study, the stool samples were collected from 130 children under 10 years old with acute diarrhea from children's hospitals in Tehran city. Genomic DNA was extracted from stool samples and was tested for the presence of DNA of T. whipplei using the SYBR Green Real-time PCR method. Positive T. whipplei samples were finally confirmed by PCR Product sequencing.

Results: The mean age of participants was 32.5 months, and $54.6 \%$ of children were female. Using the SYBR Green Real-time PCR, 9.23\% (12/130) of samples were positive for T. whipplei, which were confirmed by sequencing. 66.67\% of positive cases were males. The duration of diarrhea in infected children with T. whipplei (83.3\%) was significantly longer (OR: 5.93, 95\% Cl 1.24-28.22) compared to children with negative results (45.8\%). Other demographic factors and clinical signs had not a statistically significant relationship with T. whipplei infection.

Conclusions: In this study, T. whipplei was detected in stool samples of children with acute diarrhea. The results indicated that T. whipplei could be associated with childhood diarrhea in Iran. The health care system and physicians should be aware of the presence of T. whipplei infection in Iran, especially in childhood diarrhea.
\end{abstract}

Keywords: Tropheryma whipplei, Diarrheas, Whipple's Disease, Gastrointestinal Disease

\section{Background}

Tropheryma whipplei is a road shape Gram-positive bacterium that causes Whipple's disease. The human intestine is the only known natural site of this microorganism. After the colonization of T. whipplei in the intestinal

\footnotetext{
*Correspondence: dr.saberesmaeili@gmail.com

${ }^{2}$ National Reference Laboratory for Plague, Tularemia and Q Fever,

Research Centre for Emerging and Reemerging Infectious Diseases,

Pasteur Institute of Iran, Akanlu, Kabudar Ahang, Hamadan, Iran

Full list of author information is available at the end of the article
}

mucosa, it is taken up by macrophages cells and then replicates inside them [1].

T. whipplei has been detected in various biological specimens of infected people, including saliva, urine, blood, heart valve, myocardium, synovial fluid, skeletal muscle, feces, skin, lymph nodes, lung, bronchoalveolar fluid, stomach, spleen, liver, larynx, small intestine, colon, maxillary sinus, cerebrospinal fluid, and aqueous humor. T. whipplei can persist in vectors for several years, and asymptomatic carriers can become a large reservoir for infecting others [2]. Whipple's disease is mainly 
transmitted by the oral-oral or fecal-oral routes. A high prevalence of this disease was reported among people with poor quality health, sewage workers, and homeless people $[3,4]$. There are four forms of T. whipplei infection in humans, including classic Whipple disease, chronic localized infection, acute infection, and asymptomatic form [2]. The classic form of the Whipple disease is most common in men. Arthralgia, diarrhea, fatty diarrhea, loss of weight, lymphadenopathy, abdominal pain, hypoalbuminemia, and anemia are often observed as clinical symptoms of this form of Whipple's disease [5]. All the organs, in particular the eyes, heart, and central nervous system can be involved at the end stage of the disease. Local infection with T. whipplei is often without gastrointestinal involvement. However, it can be associated with encephalopathy, endocarditis, respiratory problems, lymphadenopathy, osteoarticular, and uveitis [2]. Recently, it was reported that $T$. whipplei caused acute infections, including gastroenteritis, bacteremia, or pneumonia [1]. Although diarrhea is one of the symptoms of the chronic, classic, and acute forms of T. whipplei infection, diagnosis is often difficult because there are no specific endoscopic findings, and this bacterium is not detectable by routine stool culture. The histopathology and PCR methods are the most well-known T. whipplei diagnostic approaches $[6,7]$. However, histopathological examination of the biopsy specimen is not commonly used for screening purposes because it is a costly and invasive method. In contrast, PCR can be preferred to identify T. whipplei in various specimens (e.g., stool, mucosa, and fluids), and it is a fast, specific, sensitive, and cost-effective method [2]. In addition, transmission electron microscopy is a helpful diagnostic tool for identifying both living and dead bacteria after therapy, when available [8]. Early diagnosis can help to treatment and eradication of T. whipplei in patients. However, misdiagnosis or improper treatment, which especially occurs in patients without the classic symptoms, can lead to lethal outcomes or irreversible neurological damage. Sometimes, patients with early symptoms may also be hard to treat due to the presence of bacteria in an inaccessible niche for the antibiotic, antibiotic resistance, or reinfection in late relapses after antibiotic treatment. Where combination therapy with antibiotics is helpful, immunosuppressive treatment can be a risk factor for displaying or aggravating Whipple's disease $[2,9,10]$. Diarrhea in children is a significant health threat in developing countries that is caused by various microorganisms. Recent studies in many countries have shown that $T$. whipplei is one of the infectious causes of diarrhea in children. The results of these studies showed that the prevalence of T. whipplei was more in the stool of children with diarrhea than healthy children as a control group. For instance, using the qPCR method, stool prevalence of T.whipplei was $27.5 \%$ among children with diarrhea from Ghana. That case group carried T.whipplei in their stool twice as frequently as controls without diarrhea [11]. In another case-control study, T. whipplei was identified in $15 \%$ of stool samples collected from children with gastroenteritis using the qPCR method. In addition, T. whipplei antibody was reported in the case group. None of the control cases were positive for the presence of bacterium or antibody. Interestingly, T. whipplei was not detected in the stool samples of children after recovering from diarrhea [12]. Therefore, T. whipplei should be considered as one of the potential causes of diarrhea in children. Since clinical symptoms are not sufficient for T. whipplei diarrhea diagnostic, molecular tests are essential to confirm this disease. In Iran, similar to most countries of the world, there is no comprehensive survey on the prevalence of T. whipplei, particularly in children with diarrhea. Therefore, the present study was performed to evaluate the presence of $T$. whipplei in stool samples of children with acute diarrhea using molecular methods.

\section{Materials and methods \\ Sample collection}

One hundred and thirty children under 10 years old with acute diarrhea who were referred to three hospitals in Tehran province (Bahrami Hospital, Mofid Hospital, and Children's Medical Center) from May 2018 to November 2018 were included in this cross-sectional study. The questionnaire form containing demographic, clinical, and laboratory information was completed at admission time.

Diarrhea stool samples were obtained from included children with acute diarrhea. Sampling was performed under an identical process and according to the hospital guidelines and regulations. A healthcare professional explained to the parents how to collect the stool of their children. Briefly, fresh diarrhea stools (10 gr) were collected inside a completely clean, dry, and sterile container with a tight screw lid. Urine was not mixed up with stool.

Stool samples were transferred to the laboratory under the cold chain conditions immediately. Future analysis was performed as soon as possible; otherwise, specimens were sore in -20 until the investigation.

\section{DNA extraction}

Genomic DNA was extracted from stool samples using the QIAamp DNA Stool Minikit (Qiagen, Germany), according to the manufacturer's recommendation. $220 \mathrm{mg}$ of each stool sample was used for DNA extraction.

To determine quality control of extracted DNA from stool samples as well as control DNA, the DNA absorbance at $260 \mathrm{~nm}$ and $280 \mathrm{~nm}$ was absorbed using a 
microplate spectrophotometer (Epoch bioTek, USA). Absorbance ratios of 260/280 nm around 1.8 were considered as good quality. Higher or lower values were considered as RNA or protein contamination. In addition, extracted DNAs were be run on gel agarose to determine quality.

The extracted DNA was stored at $-20{ }^{\circ} \mathrm{C}$ until the future test.

\section{Molecular detection of Tropheryma whipplei}

All extracted DNA samples were tested using SYBR Green quantitative real-time PCR (qPCR) for the diagnosis of the WiSP protein family gene of T. whipplei.

Tws1F (5'-AGAGAGATGGGGTGCAGGAC-3') and Tws1R (5'-AGCCTTTGCCAGACAGACAC-3) were used as forward and reverse primers (Metabion international, Germany), respectively. The $\mathrm{qPCR}$ mixture included $10 \mu \mathrm{l}$ RealQ Plus $2 \times$ Master Mix Green Low ROX $^{\mathrm{TM}}$ (Ampliqon, Denmark), $1 \mu \mathrm{l}$ from each primer (900 nmol), and $4 \mu \mathrm{l}$ of extracted DNA (11). The mixture was reached to $20 \mu \mathrm{l}$ with sterile distilled water. $T$. whipplei DNA was provided from IRCCS Sacro Cuore Don Calabria Hospital in Italy and applied as the positive control. Sterile distilled water was used as the negative control. The mixture was run in the Corbett 6000 RotorGene system (Corbett, Victoria, Australia) under the following temperature program: Initial denaturation step at $95{ }^{\circ} \mathrm{C}$ for $15 \mathrm{~min}$, and then 40 cycles of $95^{\circ} \mathrm{C}$ for $15 \mathrm{~s}$, $30 \mathrm{~s}$ at $60{ }^{\circ} \mathrm{C}$, and $72{ }^{\circ} \mathrm{C}$ for $30 \mathrm{~s}$. After amplification, the melting protocol was followed by raising the temperature slowly from 65 to $97{ }^{\circ} \mathrm{C}$ to differentiate the specific and non-specific amplified amplicons. The samples with suitable amplification and melting $\left(80 \pm 0.5{ }^{\circ} \mathrm{C}\right)$ curves were considered positive for T. whipplei.

To confirm T. whipplei positive samples, PCR products were sequenced using the Sanger sequencing method. Finally, The nucleotide sequences were blasted in GenBank (https://blast.ncbi.nlm.nih.gov/Blast.cgi) and compared with the deposited wips genes of T. whipplei in the GenBank.

\section{Statistical analysis}

Statistical analysis was performed using SPSS software version 18 (SPSS Inc., Chicago, IL, USA) for the data of this study. Chi-squared and Fisher exact tests were used to compare the variables. A p-value less than 0.05 was considered statistically significant.

\section{Results}

One hundred and thirty children with acute diarrhea were included in this study. The mean age of children was $32.5 \pm 31.6$ months; $45.4 \%$ were male, and $54.6 \%$ were female. The mean frequency of diarrhea was four times a day. The mean duration of diarrhea was 14.6 days, and the median was 3 days. Also, $42.3 \%$ of children had a fever, and $5.38 \%$ had an underlying disease (Table 1 ).

Extracted DNAs had good quality in 260/280 measurement (ratios around 1.8) and on gel agarose. Using the

Table 1 Demographic and clinical characteristics of children with acute diarrhea and their relationship to the prevalence of T. whipplei

\begin{tabular}{|c|c|c|c|c|}
\hline & $\begin{array}{l}\text { Negative } T \text {. whipplei } \\
\mathrm{N}=118\end{array}$ & $\begin{array}{l}\text { Positive } T \text {. whipplei } \\
\mathrm{N}=12\end{array}$ & $P$ value & OR $(95 \% \mathrm{Cl})$ \\
\hline Gender (male) & $51(43.2)$ & $8(66.7)$ & 0.12 & $2.63(0.75-9.21)$ \\
\hline Age median $\geq 32.9$ month & $41(34.7)$ & $5(41.7)$ & 0.75 & $1.34(0.40-4.49)$ \\
\hline Median times of diarrhea (days): $\geq 4$ times & $63(53.4)$ & $8(66.7)$ & 0.38 & $1.75(0.50-6.11)$ \\
\hline Median times of diarrhea (days) $\geq 3$ & $54(45.8)$ & $10(83.3)$ & $0.01^{*}$ & $5.93(1.24-28.22)$ \\
\hline Fever & $51(43.2)$ & $4(33.3)$ & 0.51 & $0.66(0.19-2.30)$ \\
\hline Stomachache & $29(24.6)$ & $5(41.7)$ & 0.20 & $2.19(0.65-7.44)$ \\
\hline Cramps & $34(28.8)$ & $1(8.3)$ & 0.18 & $0.91(0.83-1.00)$ \\
\hline Headache & $4(3.4)$ & $1(8.3)$ & 0.39 & $2.59(0.27-25.26)$ \\
\hline Vomiting & $29(24.6)$ & $4(33.3)$ & 0.50 & $1.53(0.43-5.47)$ \\
\hline History of contact with animal & $3(2.5)$ & $0(0.0)$ & 0.99 & - \\
\hline Family history of diarrhea & $10(8.5)$ & $0(0.0)$ & 0.60 & - \\
\hline Travel history & $6(5.1)$ & $0(0.0)$ & 0.99 & - \\
\hline Underlying disease & $5(4.2)$ & $2(16.7)$ & 0.13 & $4.52(0.78-26.34)$ \\
\hline Mucus in stool & $37(31.4)$ & $4(33.3)$ & 0.99 & $1.10(0.31-3.87)$ \\
\hline Blood in stool & $11(9.3)$ & $1(8.3)$ & 0.99 & $0.88(0.10-7.51)$ \\
\hline Pus in the stool & $10(8.5)$ & $1(8.3)$ & 0.99 & $0.98(0.12-8.41)$ \\
\hline WBC in stool & $24(20.3)$ & $3(25.0)$ & 0.71 & $1.31(0.33-5.20)$ \\
\hline
\end{tabular}

The symbol $\left(^{*}\right)$ indicates statistically significant between children with positive T. whipplei and children without T. whipplei infection. A p value $<0.05$ was considered significant 
SYBR Green qPCR method, T. whipplei was detected in 9.2\% (12/130). Then, the positive results were sequenced to confirm. The amplification and melt curves obtained from SYBR Green real-time PCR for Tropheryma whipplei detection based on the wips gene are shown in the Additional file 1: Figure S1.

Among patients infected with T. whipplei, 66.7\% were male, $58.3 \%$ were less than 32 months, and $83.3 \%$ had diarrhea for more than 3 days. Also, $66.7 \%$ of positive children had diarrhea more than four times a day. People with positive results (83.3\%) had significantly more diarrheal days than people with negative results (45.8\%) (OR: 5.93, 95\% CI 1.24-28.22). No correlation was found between positive results and other demographic factors and clinical signs (Table 1).

\section{Discussion}

Although T. whipplei colonization is often asymptomatic and especially common in adults, the carriers can transmit the microorganism to healthy people. Diarrhea is one of the main manifestations of $T$. whipplei infection in humans. Gastroenteritis occurs in both classic and acute forms of Whipple's disease [2]. T. whipplei is known as a causative agent of diarrhea in children in all developed and developing countries. Therefore, investigation of acute diarrhea associated with T. whipplei in Iran is one of the health priorities that physicians and health officials should be aware of the role of this bacterium in various syndromes, especially acute diarrhea in Iranian children. In the present cross-sectional study, $9.23 \%$ of collected stool samples of children with acute diarrhea referred to three hospitals in Tehran city (in Iran) during 2018 were positive for T. whipplei using the qPCR method. As far as we know, except for a recently reported study, there have not been any studies on T. whipplei in Iran so far. In that study, $10 \%$ of immunocompromised children in a province (Qom) in Iran had T. whipplei DNA (using qPCR) in their stool samples [13]. Since T. whipplei is a hard-growing bacterium, bacterial culture requires a long incubation time that is not available in many laboratories. Therefore, molecular methods are a preferred method to identify T. whipplei infection in patients [14, 15]. For instance, qPCR is a beneficial method for firstline screening of T. whipplei in stool specimens [16].

This study shows that the prevalence of T. whipplei is much lower than similar studies conducted in other countries, including Ghana 27.5\% [11], France 15\% [12], Gabon 40\% [17], Laos 48\% [18], and Senegal 31.2\% [19]. Various factors, such as differences in health care systems and climatic conditions, could be involved in incidence variations between different regions in the world. The prevalence of diarrhea caused by $T$. whipplei in children is significantly higher in tropical regions, particularly in countries with low levels of health $[2,5]$. In the present study, there was no association between T. whipplei infection with history of travel, contact with animals, family history of diarrhea, and a set of clinical and laboratory findings. However, diarrhea for $\geq 3$ days was observed among $83.3 \%$ of children with T. whipplei infection. In contrast, only $45.8 \%$ of children with negative T. whipplei experienced prolonged diarrhea. According to our results, it seems that $T$. whipplei might cause prolonged diarrhea compared to previously known bacterial agents causing diarrhea.

Since T. whipplei is an unknown causative agent of acute diarrhea in children among physicians and laboratories in Iran, our results, even with this reported prevalence, can be a warning to inform physicians and laboratories of the existence of this disease in Iran. Considering that $T$. whipplei may colonize all ages asymptomatically, future epidemiological studies are required to assay the association of T. whipplei with acute diarrhea in children in different parts of the world, including Iran. In addition, diagnosis, control, and treatment of $T$. whipplei should be considered by health systems in Iran and even other countries.

One of the limitations of the study is the small number of samples. Future studies among a larger population with different demographic and clinical characteristics can determine comprehensive knowledge on the frequency of T. whipplei in patients with diarrhea. In addition, this study did not include a control group (children without diarrhea) which made result interpretation more difficult. Another limitation was that we did not measure possible antibodies produced against $T$. whipplei. Furthermore, no other clinical microbiology diagnosis was performed to identify other enteric pathogens in positive samples of T. whipplei. These data could help to interpret of obtaining results in the present studies.

\section{Conclusions}

In this study, the stool prevalence of T. whipplei among children with acute diarrhea in Iran was $9.23 \%$ using the qPCR method. Significantly, children with $T$. whipplei infectious diarrhea had prolonged diarrhea than children without T. whipplei infection (83.3\% vs. $45.8 \%)$. Therefore, T. whipplei could be a potential cause of acute diarrhea in children in Iran, and physicians and laboratories should be aware of the presence of this disease in Iran. However, comprehensive studies are required to evaluate the epidemiology and pathogenicity of T. whipplei in pediatric diarrhea in Iran. This study showed the importance of qPCR in the diagnosis of T. whipplei infection as a possible cause of diarrhea in children. Therefore, molecular diagnosis of T. whipplei is recommended, especially when diarrhea lasts more than 3-4 days. 


\section{Abbreviations}

T. whipplei: Tropheryma whipplei; qPCR: Quantitative real-time PCR.

\section{Supplementary Information}

The online version contains supplementary material available at https://doi. org/10.1186/s12879-022-07198-5.

Additional file 1: Figure S1. Representative the amplification plot (A) and melting curve (B) of SYBR Green real-time PCR for Tropheryma whipplei detection based on the wips gene in specimens. Positive and negative controls were run in all runs. A) The cycle number is shown on the $x$-axis and change in fluorescent intensity is shown on the $y$-axis. B) Positive control and samples have a melt pick in $80 \pm 0.5^{\circ} \mathrm{C}$ in melting curves.

\section{Acknowledgements}

The authors are thankful for the cooperation of Children's Medical Center, Bahrami, and Mofid hospitals as well as Tarbiat Modares University as their help in sampling.

\section{Authors' contributions}

$\mathrm{SE}$, SS, and AE helped in the conception and design of the study. SE, BB, ML, and FBA contributed to data collection. SE, PE, NB, and ML helped in the first draft writing. SE, BB, SS, and NB did the final draft. All authors contributed to the final approval of the version to be submitted. All authors read and approved the final manuscript

\section{Funding}

This study was financially supported by the Pediatric Infectious Diseases Research Center of Iran University of Medical Sciences (Grant No. 98-3-4916054) and the Pasteur Institute of Iran.

\section{Availability of data and materials}

The datasets used and/or analyzed during the current study are available from the corresponding author on reasonable request.

\section{Declarations}

\section{Ethics approval and consent to participate}

This study was approved by the Ethics Committee of Iran University of Medical Sciences with a committee's reference number "IR.IUMS.REC.1398.1103". All methods and instructions were performed under institutional guidelines and regulations. For sampling and completing the questionnaire, informed consent was obtained from all the parents of included children with the inscribed and signed consent.

\section{Consent for publication}

Not applicable.

\section{Competing interests}

The authors declare that they have no competing interests.

\section{Author details}

${ }^{1}$ Research Center of Pediatric Infectious Diseases, Institute of Immunology and Infectious Diseases, Iran University of Medical Sciences, Tehran, Iran. ${ }^{2}$ National Reference Laboratory for Plague, Tularemia and Q Fever, Research Centre for Emerging and Reemerging Infectious Diseases, Pasteur Institute of Iran, Akanlu, Kabudar Ahang, Hamadan, Iran. ${ }^{3}$ Department of Epidemiology and Biostatistics, Research Centre for Emerging and Reemerging Infectious Diseases, Pasteur Institute of Iran, Tehran, Iran. ${ }^{4}$ Department of Bacteriology, Faculty of Medical Sciences, Tarbiat Modares University, Tehran, Iran.

\section{References}

1. Lagier JC, Fenollar F, Raoult D. Acute infections caused by Tropheryma whipplei. Future Microbiol. 2017;12:247-54.

2. Dolmans RA, Boel CE, Lacle MM, Kusters JG. Clinical manifestations, treatment, and diagnosis of Tropheryma whipplei infections. Clin Microbiol Rev. 2017;30(2):529.

3. Fenollar F, Trani M, Davoust B, Salle B, Birg M-L, Rolain J-M, Raoult D. Prevalence of asymptomatic Tropheryma whipplei carriage among humans and nonhuman primates. J Infect Dis. 2008:197(6):880-7.

4. Brouqui P, Badiaga S, Benkouiten S, Ratmanov P, Raoult D, Fenollar F. Tropheryma whipplei prevalence strongly suggests human transmission in homeless shelters. Int J Infect Dis. 2013;17(1):e67-8.

5. Fenollar F, Lagier J-C, Raoult D. Tropheryma whipplei and Whipple's disease. J Infect. 2014;69(2):103-12.

6. Edouard S, Luciani L, Lagier J-C, Raoult D. Current knowledge for the microbiological diagnosis of Tropheryma whipplei infection. Expert Opin Orphan Drugs. 2020;8(7):237-44.

7. Lagier J-C, Raoult D. Whipple's disease and Tropheryma whipplei infections: when to suspect them and how to diagnose and treat them. Curr Opin Infect Dis. 2018;31(6):463-70.

8. Loiodice A, Losurdo G, lannone A, Rossi R, Fiore MG, Piscitelli D. Transmission electron microscopy helpfulness in Whipple's disease masked by immunosuppressant therapy for arthritis. APMIS. 2018;126(1):92-6.

9. Melas N, Amin R, Gyllemark P, Younes AH, Almer S. Whipple's disease: the great masquerader - a high level of suspicion is the key to diagnosis. BMC Gastroenterol. 2021;21(1):1-8.

10. Schneider T, Moos V, Loddenkemper C, Marth T, Fenollar F, Raoult D. Whipple's disease: new aspects of pathogenesis and treatment. Lancet Infect Dis. 2008;8(3):179-90.

11. Vinnemeier CD, Klupp EM, Krumkamp R, Rolling T, Fischer N, Owusu-Dabo E, Addo MM, Adu-Sarkodie Y, Käsmaier J, Aepfelbacher M, et al. Tropheryma whipplei in children with diarrhoea in rural Ghana. Clin Microbiol Infect. 2016;22(1):65 e61-65 e63.

12. Raoult D, Fenollar F, Rolain J-M, Minodier P, Bosdure E, Li W, Garnier J-M, Richet H. Tropheryma whipplei in children with gastroenteritis. Emerg Infect Dis. 2010;16(5):776.

13. Shams S, Rezaie N, Beltrame A, Moro L, Piubelli C, Amiri FB, Esmaeili S. Tropheryma whipplei intestinal colonization in immunocompromised children in Iran: a preliminary study. Future Microbiol. 2021;16(15):1161-6.

14. Fenollar F, Ponge T, La Scola B, Lagier JC, Lefebvre M, Raoult D. First isolation of Tropheryma whipplei from bronchoalveolar fluid and clinical implications. J Infect. 2012:65(3):275-8.

15. Raoult D, Birg ML, La Scola B, Fournier PE, Enea M, Lepidi H, Roux V, Piette JC, Vandenesch F, Vital-Durand D, et al. Cultivation of the bacillus of Whipple's disease. N Engl J Med. 2000;342(9):620-5.

16. Fenollar F, Laouira S, Lepidi H, Rolain J-M, Raoult D. Value of Tropheryma whipplei quantitative polymerase chain reaction assay for the diagnosis of Whipple disease: usefulness of saliva and stool specimens for first-line screening. Clin Infect Dis. 2008;47(5):659-67.

17. Ramharter M, Harrison N, Bühler T, Herold B, Lagler H, Lötsch F, MomboNgoma G, Müller C, Adegnika A, Kremsner P. Prevalence and risk factor assessment of Tropheryma whipplei in a rural community in Gabon: a community-based cross-sectional study. Clin Microbiol Infect. 2014;20(11):1189-94.

18. Dubot-Pérès A, Phommasone K, Sibounheuang B, Vongsouvath M, Mayxay M, Raoult D, Newton PN, Fenollar F. High prevalence of Tropheryma whipplei in Lao kindergarten children. PLoS Negl Trop Dis. 2015;9(2):e0003538.

19. Bassene H, Tall A, Sokhna C, Ratmanov P, Trape J-F, Raoult D, Fenollar F. Tropheryma whipplei: a common bacterium in rural Senegal. PLoS Negl Trop Dis. 2011;5(12):e1403.

\section{Publisher's Note}

Springer Nature remains neutral with regard to jurisdictional claims in published maps and institutional affiliations.

Received: 22 September 2021 Accepted: 23 February 2022

Published online: 27 February 2022 